\title{
Líneas metodológicas para la formación de profesores de lenguas extranjeras
}

\author{
Marta Higueras García \\ Instituto Cervantes \\ martahig@cervantes.es
}

Resumen: La formación de profesores de lenguas extranjeras debe responder a la complejidad de la profesión, ya que para ser un buen profesional de la enseñanza en este campo se requiere una serie de conocimientos sobre la lengua, sobre su didáctica, sobre adquisición de lenguas, sobre técnicas para enseñar distintos contenidos lingüísticos, etc., además de una serie de habilidades docentes, comunicativas o interculturales que solo pueden ejercitarse y mejorarse a través de la práctica. Como consecuencia, «el qué» de los cursos de formación de profesores $\mathrm{y}$ «el cómo» deben enfocarse las actividades formativas no son preguntas a las que se pueda responder de forma sencilla. En este artículo expondremos algunas líneas metodológicas que aseguren una formación de calidad en el enfoque en que nos situamos; incidiremos en qué enseñar y en cómo hacerlo, atendiendo tanto a la dimensión del profesor como docente, como a la del profesor como miembro de un centro educativo y de una comunidad profesional. Se explicará cómo se diseñó, se desarrolló y se evaluó el taller que tuvo lugar en el marco de las primeras jornadas EnELE celebradas en la Universitat Rovira i Virgili y finalizaremos con el comentario de algunos ejemplos de actividades formativas realizadas en el Instituto Cervantes (IC). ${ }^{1}$

Palabras claveः Formación de profesores, líneas metodológicas, contenidos de los cursos de formación de profesores, metodología de los cursos de formación de profesores, calidad, actividades de formación de profesores en el Instituto Cervantes.

1. Agradezco a mis colegas del Departamento de Formación de profesores del Instituto Cervantes las sugerencias y comentarios aportados a este artículo. 


\begin{abstract}
The training of teachers of foreign languages needs to respond to the complexity of the profession. To be a good professional in this field, trainees need knowledge about language, didactics, language acquisition, techniques to teach different linguistic contents, etc. In addition, a series of didactic, communicative and intercultural skills are required. These can only be improved through constant practice. Consequently, «what» to include in teacher training courses and «how» to address training activities is not easy to know. This article will explain some methodological lines that guarantee quality in training within our approach: what to teach and how to do it, taking into account the role of teachers in their teaching activity, and as members of the educational centre and professional community. It will explain in detail how the workshop that took place in the first EnELE Congress (Rovira i Virgili University) was designed, developed and assessed. Finally, this article will comment on training activities held in Instituto Cervantes (IC).
\end{abstract}

Keywords: Teacher training, methodological lines, contents in teacher training courses, Methodology of teacher training courses, Quality, Teacher training activities at Instituto Cervantes. 


\section{El qué de la formación de profesores de lenguas extranjeras}

Antes de profundizar en lo específico de nuestra profesión, puede ser pertinente comenzar por reflexionar sobre qué es enseñar: ¿es llenar de conocimientos las mentes de los alumnos (the jug and mug view of knowledge transmission)?, ¿̇es conseguir que el alumno desarrolle habilidades y mejore ciertas actitudes?, ¿consiste tal vez en desarrollar la autonomía? Algunas de las respuestas de los asistentes al taller, como podemos leer en la tabla de la figura 1, pusieron de manifiesto que la visión más tradicional de la transmisión de conocimientos está prácticamente superada:

«Acompañar»; «Entender las necesidades de los alumnos»; «Ser entrenador, un míster, que saca lo mejor de cada jugador para formar equipo»; «Orientar, guiar, acompañar, supervisar»; «Sacar, extraer lo que el alumno tiene dentro»; «Dar herramientas para progresar»; «Despertar la curiosidad y crear motivación por el aprendizaje de la lengua. El alumno es activo en este proceso. El profesor es catalizador»; «Ser vehículo de adquisición de destrezas y competencias. El profesor es una brújula, que orienta y da alas y herramientas de trabajo al alumno».

Figura 1.

Más allá de la discusión acerca de las disciplinas implicadas en la formación del profesor de español (la lingüística teórica y aplicada, la didáctica de lenguas extranjeras y otras ciencias afines ${ }^{2}$ ) y de la necesidad de una formación específica en este ámbito, aspectos que ya han sido debatidos ampliamente en otros escritos y por otros autores, queremos aportar en estas páginas una reflexión que parte de la observación de la profesión y de lo que el profesor hace o debería ser capaz de hacer, para describir, a partir de esos listados de acciones que realiza un docente competente, en qué consiste ser un buen profesor de lenguas extranjeras. Según Verdía (2010: 60) y Perrenoud (2001, 2004), hubo una primera etapa de la formación de profesores que estuvo muy centrada en la transmisión de conocimientos teóricos, seguida de otra que puso el énfasis en las habilidades, sin que resultara todavía fácil que el profesor en formación (en adelante $\mathrm{PeF}$ ) pudiera transferir lo aprendido a su realidad de aula. En el momento actual, en que se

2. Esta formación debe ser multidisciplinar y requiere una sólida preparación y un profundo conocimiento de la lengua y la cultura, pero no solo de ellos: basta con analizar los trece inventarios del Plan curricular del Instituto Cervantes (Instituto Cervantes, 2006) para comprobar que desarrollar la competencia comunicativa de los alumnos en su descripción actual, heredera de la concepción del MCER (Consejo de Europa, 2001), implica dominar tanto una perspectiva lingüística e intercultural, como la dimensión de procedimientos de aprendizaje. 
cuestiona la eficacia de la asistencia a talleres como única vía de desarrollo profesional y se busca cómo mejorar el desempeño de estos docentes, se ha puesto el foco en partir de las acciones que lleva a cabo el profesor competente, para describirlas y conocer qué competencias precisa desarrollar para realizarlas eficientemente. Para Verdía (2010:61), estas tareas se agruparían en las siguientes áreas, que responden a las tres dimensiones del PeF: como docente, como profesional y como aprendiente.

+ Planificación y organización del proceso de enseñanza y aprendizaje.

+ Gestión del proceso de enseñanza y aprendizaje.

- Evaluación del proceso de enseñanza y aprendizaje.

- Integración en un equipo docente y en un centro de enseñanza.

- Reflexión crítica sobre el proceso de enseñanza y aprendizaje y sobre el desarrollo profesional.

La decisión de clasificar las tareas que realiza el profesor competente en áreas o ámbitos formativos ${ }^{3}$ implica una nueva visión de la formación de profesores y ha permitido diseñar una oferta formativa en el IC que atiende a todas las dimensiones de la profesión. Consecuentemente, como el énfasis se pone en qué sabe y en qué sabe hacer un PeF en cada etapa de su desarrollo profesional, los cursos se pueden dividir por niveles formativos, lo que permite mejorar el aprovechamiento de los asistentes. ${ }^{4}$

Para ir más allá en este planteamiento de la formación de profesores de lenguas extranjeras, es preciso profundizar en el concepto de «competencia docente», con el que nos referimos a un conjunto de componentes interrelacionados y articulados entre sí. No se puede hablar de competencia sin unos conocimientos sobre la materia, sin el desarrollo de unas habilidades, sin unas actitudes hacia la tarea que realizamos y sin una capacidad de aprender a lo largo de toda la carrera profesional. En el anexo 1 se muestra la tabla con la que trabajaron los asistentes, que recoge la visión de la competencia que se manejaba hace unos años, en el documento interno e inédito del Marco general del Instituto Cervantes para la formación de profesores de español como lengua extranjera y como segunda lengua (2004). Dicha actividad sirvió para que los $\mathrm{PeF}$ que asistieron al taller tuvieran una visión de conjunto y también para que anotaran los conocimientos, habilidades y actitudes con los que ya contaban, a pesar de estar en los primeros momentos de su

3. Desde el programa de formación del año 2013, sin embargo, a raíz de la publicación del documento Las competencias clave del profesorado de lenguas segundas y extranjeras, (IC, 2012), en lugar de hablar de ámbitos formativos se utiliza el término «competencias». (Véase anexo 2.)

4. Desde el año 2009, la oferta formativa del IC para profesores de lenguas extranjeras se ofrece en tres niveles de desarrollo profesional (A, B y C), lo que ha permitido ofrecer cursos homogéneos y ha sido muy bien aceptado y valorado tanto por los formadores como por los PeF. 
desarrollo profesional. A pesar de que esta definición ha permitido ampliar los horizontes de la formación, tiene el inconveniente de que se describe como una acumulación de recursos que debería tener el profesor para llegar a ser competente; además, poco podía aportar sobre la actuación del profesor. El que tengamos conocimientos adquiridos, habilidades desarrolladas y actitudes determinadas no significa que seamos competentes, no es suficiente. Tener esos recursos no garantiza la competencia profesional. La evolución del concepto de competencia docente nos permite afirmar que no se trata de hablar de competencias ni de tener competencias, sino de actuar con competencia, ${ }^{5}$ tanto en el aula como fuera de ella, con otros colegas. Esta es la visión que se desprende de la definición de competencia docente de Perrenoud, en la que ser competente se identifica con ser capaz de actuar y de tener éxito en una actividad, movilizando una combinación apropiada de recursos; en otras palabras, se enfatiza la idea de que solo se puede medir la competencia en la actuación:

Competencia es la aptitud para enfrentarse eficazmente a una familia de situaciones análogas, movilizando a conciencia y de manera a la vez rápida, pertinente y creativa, recursos cognitivos, saberes, capacidades, microcompetencias, informaciones, valores, actitudes, esquemas de percepción, de evaluación y de razonamiento (Perrenoud 2001:509).

Esta habilidad de saber movilizar los recursos necesarios para resolver las tareas a las que se enfrenta el profesional en una determinada situación (saber actuar) solo se desarrolla en la experiencia, actuando en la práctica; de ahí que un principio metodológico esencial para ofrecer formación de calidad es partir de la experiencia del alumno y de su realidad y volver a ella, es decir, garantizar que hay una transferencia de lo aprendido al contexto del PeF, para poder contrastar ese progreso en la competencia docente, como defiende el enfoque realista.

Tras aclarar estos conceptos y para profundizar en las consecuencias que se derivan de esta concepción de la formación de profesores, se presentaron dos proyectos en los que participa actualmente el IC. El primero de ellos es el informe de investigación Qué es ser un buen profesor o una buena profesora del Instituto Cervantes, ${ }^{6}$ expuesto ya en el congreso COMPROFES (VV.AA. 2012), que ha permitido profundizar en las creencias de distintos profesionales de la institución sobre el perfil del profesor competente, como paso previo para determinar las

5. Para una revisión del concepto de competencia y sus características, véase también Rodrigo (2011) y Martín Peris (2009), quien defiende que la competencia del profesor de ELE o EL2 está integrada por una competencia general, común a todas las materias y disciplinas, y unos conocimientos específicos de las disciplinas implicadas, que resume en cuatro aspectos: lengua, cultura y comunicación; adquisición y proceso de aprendizaje de ELE y EL2; fundamentos y prácticas didácticas en ELE y EL2; y metodología de la enseñanza, didáctica de la lengua y evaluación.

6. El informe puede consultarse en <http://cfp.cervantes.es/recursos/proyectos/default.htm>. 
competencias clave para este colectivo de profesores. El proyecto se desarrolló en dos fases: en la primera, se consultó a 39 participantes (directivos, técnicos, expertos externos, responsables de recursos humanos, formadores de profesores, jefes de estudio, etc.) a través de la herramienta de investigación cualitativa de los grupos de discusión, dirigida por el psicólogo y sociólogo Fernando Cembranos; en una segunda fase, se consultó también a profesores y alumnos del IC (en total, 575 de 48 centros distintos). Las conclusiones que se desprenden de dicha investigación son que, para el grupo de personas consultadas, un buen profesor o una buena profesora de lenguas extranjeras reúne las siguientes características, en este orden de importancia:

+ tiene una buena formación,

+ promueve una enseñanza centrada en el alumno,

- gestiona bien el proceso de aprendizaje,

- tiene un buen núcleo emocional,

- se preocupa por su desarrollo profesional,

+ trabaja en equipo,

- tiene una buena capacidad para relacionarse y

+ se compromete con la institución.

En segundo lugar, se presentó someramente en la sesión el proyecto European Profiling Grid (VV.AA. 2011-2013)7 y se animó a todos los asistentes a participar en el pilotaje de la parrilla de criterios que permitirán describir al profesor de lenguas extranjeras competente, partiendo también de lo que sabe y es capaz de hacer en su entorno profesional. Se trata, pues, de un proyecto que se enmarca en esa nueva concepción de la competencia docente que acabamos de presentar. En el proyecto EPG se realizará un pilotaje a escala europea de la versión corregida y ampliada de la parrilla inicial (North et alii 2007-2009, Rossner 2010), que pretendía describir en seis niveles lo que debía saber y lo que debía saber hacer un profesor de lenguas extranjeras, sirviéndose de cuatro parámetros o categorías: lengua, cualificaciones, competencias generales y destrezas complementarias. La parrilla se disenó con el propósito de servir como instrumento al servicio de la transparencia de las titulaciones, que permitiera la evaluación de las competencias de los profesores de lenguas y que fuera útil tanto a profesores de lenguas como a formadores de profesores, responsables académicos y personal de recursos humanos. Se caracteriza por ser genérica, ya que es aplicable a diferentes contextos europeos; polivalente, dado

7. Se trata de un proyecto Leonardo, de transferencia de innovación, cofinanciado por la Comisión Europea, que se inició en 2011 y finalizará en 2013, con la publicación de la nueva versión de la parrilla. En este proyecto colaboran once instituciones europeas de prestigio: CIEP, EAQUALS, British Council, Instituto Cervantes, BAQLS OPTIMA, Goethe-Institute, CEBS, ELS-Bell Education Ltd, UNISTRASI, HvA y SU. Puede ampliarse la información sobre este proyecto y sobre cómo participar en él en $<$ http://www.epg-project.eu/ $>$. 
que contará con una versión en papel y otra electrónica, y compatible con otros proyectos europeos (como MCER y Europass). La versión actual cuenta con trece categorías y 73 descriptores, aunque es posible que esta cifra varíe con el desarrollo del pilotaje. En 2013, como resultado de dicho proyecto interinstitucional, se publicará la parrilla validada y una guía para su utilización.

Tanto el concepto ampliado de la competencia docente como los dos proyectos presentados ofrecen una visión completa, desde la actuación, de cuáles deberían ser los objetivos y los contenidos de la formación de profesores de lenguas extranjeras, sobre todo en el contexto europeo, y de la necesidad de intercalar teoría y práctica docente desde el inicio de la formación (Esteve 2004).

\section{El cómo de la formación de profesores de lenguas extranjeras}

Para ofrecer una formación innovadora y de calidad, desde nuestro punto de vista, además de integrar ese nuevo concepto de competencia docente, es preciso incorporar una metodología que permita diseñar actividades formativas que motiven a los $\mathrm{PeF}$, que respondan a sus necesidades y que tengan en cuenta lo que la investigación en formación de profesores ha aportado en estas últimas décadas. Dos documentos esenciales son el EPLTE (Kelly et alii 2004) ${ }^{8}$ y el QuailiTraining (Muresan et alii 2007). ${ }^{9} \mathrm{El}$ primero - conocido en la comunidad de profesores como el Profile - constituye un marco de referencia para los planes formativos de profesores de lenguas extranjeras; presenta 41 ítems que describen los elementos esenciales que pueden incluirse en los programas de formación de profesores para dotar a los profesionales de la enseñanza de las habilidades y conocimientos necesarios, así como de otras competencias profesionales. Tanto el programa máster del IC, desarrollado en colaboración con la UIMP, como las actividades formativas que se realizan en el IC se basan en sus propuestas.

Se detallan a continuación algunos de esos principios metodológicos, que están íntimamente interrelacionados y que se basan, en su mayoría, en el enfoque humanístico y en el constructivismo. El primero de ellos se preocupa por tratar al aprendiente como persona, con una implicación global en el aprendizaje, en lugar de centrarse solo en las destrezas cognitivas, y considera que el aprendizaje significativo se lleva a cabo únicamente cuando lo enseñado tiene relevancia personal para el alumno, implica una participación activa y el aprendizaje se basa en la expe-

8. The European Profile for Language Teacher Education - A Frame of Reference (Kelly et alii 2004). Veáse también Kelly (2012).

9. QualiTraining - A Training Guide for Quality Assurance in Language Education (Muresan et alii 2007). Véase también Muresan (2012). 
riencia. En cuanto al constructivismo, defiende que se aprende reestructurando los conocimientos previos y partiendo de las estructuras cognitivas preestablecidas.

\subsection{Aprender a través de la acción}

La idea procede de Piaget, quien afirmaba que no tiene sentido proporcionar contenidos acabados, sino que el aprendiente ha de llegar a ellos mediante la experimentación, razón por la que su teoría de aprendizaje se conoce como aprendizaje por acción. De acuerdo con este principio, que nosotros aplicamos ahora a la formación de profesores, el PeF, al igual que el alumno en la clase de lenguas extranjeras, debe tener un papel activo (Matei et alii 2007:9-10), realizar acciones, y no recibir pasivamente los contenidos. Retomando el concepto de competencia docente presentado más arriba, recordemos que es precisamente al realizar acciones cuando debemos demostrar que tenemos conocimientos, habilidades y actitudes y, sobre todo, que las movilizamos adecuadamente. En otras palabras, se puede decir que tanto el alumno de LE como el PeF aprenden y tienen competencia - comunicativa o docente, respectivamente-cuando saben actuar en situaciones determinadas. ${ }^{10}$ Las competencias solo se pueden evaluar en la actuación; por eso en una buena actividad formativa que siga este enfoque debería haber actividades de teoría y de práctica docente intercaladas e integradas. Abundamos en la idea de que las competencias profesionales se desarrollan a través de la formación, pero no solo con ella, sino también en el día a día de la práctica, en situaciones de trabajo reales en las que se pueda evaluar al profesor en acción.

\subsection{Facilitar la interacción entre los participantes}

La interacción que conduce al aprendizaje solo tiene lugar si existe una buena moderación o «facilitación» ${ }^{11}$ por parte del formador de profesores, si este ha

10. Perrenoud (2001: 508-523) analiza cómo debe ser la formación de profesores de calidad y presenta diez criterios, el primero de los cuales es precisamente articular un plan de formación en torno a competencias. No debemos olvidar que las competencias se pueden adquirir, aprender o desarrollar, es decir, que un profesional puede aprender a ser competente; y que además, si no se ejercitan, las competencias se pueden perder. Por todo ello son un componente esencial en la formación de profesores.

11. A la pregunta de qué es facilitar, el grupo de PeF del curso Primeros pasos en la formación de profesores (edición de 2012: <http://cfp.cervantes.es/actividades_formativas/cursos/cursos_cervantes/CFP292-12.htm>) respondió lo siguiente: «[...] es moderar, ayudar, guiar, superar dificultades, activar conocimiento previo, es proporcionar estrategias y apoyo emocional, es crear puentes entre los $\mathrm{PeF}$ y el conocimiento, es responder a las necesidades de los $\mathrm{PeF}$ ». En cuanto a los desafíos que presenta la «facilitación», en ese mismo taller se destacaron los siguientes: «puede que un participante domine la conversación o que haya un participante «sabelotodo»; el formador tendrá que filtrar las contribuciones en función de si son de ayuda o no para el objetivo del debate; tendrá que tomar decisiones sobre el camino que debe tomar el debate; tendrá que decidir cuándo va a dar su opinión, sintetizar las opiniones y resumirlas; es preciso que el formador implique a todos los $\mathrm{PeF}$ (especialmente a los más callados) y también debe recordar las contribuciones individuales y mostrar su lado amable en todo momento». 
desarrollado esa habilidad (Bolitho y Wright 1997). Ser un buen «facilitador» supone permitir que los participantes trabajen juntos, que resuelvan en grupo los retos que haya propuesto el formador o el profesor, con sus conocimientos previos y la reflexión conjunta. Esto implica que la persona que dirige una actividad - formativa o para el aprendizaje de la lengua - tiene que animar a los asistentes a que se expresen, para que encuentren sus propios papeles, valores y creencias, pero también tiene que permitir que haya periodos de silencio, que propicien la reflexión y que les permitan conceptualizar lo aprendido (Matei et alii 2007: 9-10). Subyace aquí el enfoque del interaccionismo social, defendido por Vigotsky, según el cual las personas aprenden y dan sentido al mundo que les rodea a través de las interacciones en las que participan desde el momento en el que nacen. Según esta teoría, cuando el aprendiente trabaja con otra persona que tiene un nivel algo superior al suyo, gracias al proceso de mediación, consigue resolver la tarea y aprender. Por lo tanto, se deben diseñar actividades que potencien esa construcción social del conocimiento, a través de la reflexión sobre la propia aportación y la de los demás, en actividades grupales que permitan la interacción. También el aprendizaje realista insiste en este aspecto. Como resume Rodrigo (2011: 55), «en el aprendizaje realista, la interacción se entiende como comunicarse para aprender: la reflexión individual se enriquece con la interacción mutua entre los miembros de la comunidad de aprendizaje. Esta interacción conduce a cada persona a una comparación: entre uno mismo y los demás; consigo mismo; entre práctica, teoría y teoría y comparación en el tiempo».

Para que se produzca esa «facilitación» es preciso que las actividades se hayan diseñado para estimular el debate, que haya un ambiente relajado y propicio entre los participantes, que todos crean que los grupos de personas pueden pensar mejor que los individuos aisladamente y que el formador o profesor de lenguas extranjeras haya desarrollado esa habilidad; de ahí que sea preferible que la formación que reciba este último se haga mediante talleres y no mediante conferencias, pues, si aprende a trabajar en grupo, podrá moderar $\mathrm{y}$ «elicitar» conocimiento de sus alumnos cuando enseñe lenguas extranjeras.

\subsection{Transferir el aprendizaje al contexto del participante}

Se trata de crear vínculos entre la teoría con minúscula ${ }^{12}$ y el mundo real del participante, de que el PeF descubra información que es relevante para él y su

12. Korthagen (2001) distingue entre el conocimiento conceptual (Teoría con mayúscula) y el perceptual (teoría con minúscula). El segundo equivale a la conceptualización que puede llegar a elaborar el propio sujeto en formación a partir de la reflexión sobre sus experiencias y que progresivamente se va contrastando con el saber teórico más elaborado. A partir de esta contraposición, que nace del propio sujeto y tiene lugar en él, se construye un nuevo conocimiento que se convierte a su vez en nuevo objeto de reflexión. Se inician así una serie 
contexto, no la información que ha decidido impartir previamente el formador. Si una actividad se basa en la práctica docente y parte del contexto del PeF, será mucho más fácil que esa transferencia tenga lugar.

\subsection{Potenciar la introspección y la reflexión}

Los programas formativos deben potenciar la reflexión y el análisis (sobre materiales de enseñanza, producciones o la actuación del profesor, documentos curriculares, obras de referencia, creencias, etc.); además, como acabamos de ver, esa reflexión debería partir de los conocimientos previos del $\mathrm{PeF}$, para que este vaya construyendo su teoría con minúscula. El enfoque reflexivo aporta una serie de instrumentos para la reflexión sistemática, como portfolios, diarios, etc, que pueden ayudar al profesor en este proceso introspectivo y que deberían estar muy presentes en las actividades formativas. Por eso se explicó en el taller la importancia de completar un portfolio o un diario del profesor.

\subsection{Fomentar la autonomía}

Además de esa introspección y reflexión, conviene que las actividades formativas fomenten la autonomía de los PeF, entendida como autorregulación: el PeF establece su punto de partida, traza su itinerario y sus objetivos a medio y corto plazo y toma decisiones para llevar a cabo el plan que ha diseñado. Por ello, en el taller se explicó la estructura y la finalidad del Portfolio europeo para futuros profesores de idiomas (PEFPI) (Newby et alii 2007). ${ }^{13}$ Este documento consta de tres secciones: declaración personal, autoevaluación con siete categorías y 193 descriptores de la competencia docente y, finalmente, el dosier. Su principal finalidad es promover la reflexión sobre la competencia docente y servir como instrumento de autoevaluación, así como registrar gráficamente las experiencias de enseñanza de los PeF y su progreso a lo largo de su proceso formativo.

\subsection{Buscar el cambio}

Se debe promover el cambio (Bolitho y Wright 1997) en las prácticas, los métodos y los enfoques, para lo cual es necesaria la introspección que sugiere el enfoque reflexivo, para ser consciente de lo que hacemos $y$, sobre todo, de por qué lo hacemos, y así podamos contrastar nuestra actuación con la teoría y con la actuación de otros compañeros.

de procesos cíclicos, en los cuales los nuevos conocimientos entran en conexión con saberes ya interiorizados y construidos por el mismo sujeto.

13. En inglés se denomina EPOSTL. Véase también Komorowska (2012). 


\subsection{Garantizar la coherencia entre los distintos componentes y fases de la actividad formativa}

Dicho cambio, que deseamos que se produzca en el PeF, será más patente si existe coherencia entre los distintos componentes de la actividad formativa (objetivos, contenidos, metodología y evaluación) y, también, si existe coherencia entre el diseño de la actividad formativa, su desarrollo y su revisión y evaluación. Todo debe responder al mismo enfoque: una formación que potencia las habilidades, la reflexión y la mejora de la actuación de los profesores no podría concluir, por ejemplo, con una evaluación únicamente de conocimientos, sino con una experiencia docente que permita contrastar el progreso del PeF y en la que este se plantee nuevos interrogantes, para abrir un nuevo ciclo reflexivo. También es de suma importancia la coherencia entre la metodología que se está explicando y la metodología que adopte el formador; como nos recuerdan Bolitho y Wright (1997), «las ideas del formador pueden ser útiles o no para los participantes; sin embargo, las habilidades del formador son vitales para el proceso de aprendizaje de los participantes».

\subsection{Responder a las necesidades del PeF y comenzar desde el punto en el que se encuentra}

Este principio que resulta tan obvio en la enseñanza de lenguas extranjeras, y que casi todos los profesores aplican, suele ser más infrecuente en las actividades formativas, ya que muchas veces están prácticamente cerradas y constreñidas a unos materiales enviados de antemano y, además, suelen contar con un tiempo muy limitado, que impiden que se pueda negociar sobre qué necesitan aprender los PeF. Matei et alii (2007) lo resumen en el siguiente principio: hay que empezar en el punto en que se encuentra el alumno. Realizar un análisis de necesidades es un paso fundamental para el diseño de una actividad formativa, el segundo de la propuesta de Bernaus (2012, basado en Thorne y Mackey 2003):

\begin{tabular}{|c|c|l|}
\hline \multirow{2}{*}{ Antes de la formación } & 1 & Idea \\
\cline { 2 - 3 } & 2 & Análisis de necesidades \\
\cline { 2 - 3 } & 3 & Diseño del curso \\
\hline & 4 & Desarrollo de los materiales \\
\hline Durante la formación & 5 & Desarrollo del curso y «facilitación» \\
\hline Después de la formación & 6 & Revisión y evaluación \\
\hline
\end{tabular}

Figura 2. 
Para concluir este epígrafe, insistimos en que deberíamos alejarnos de modelos formativos que se limitan a la mera transmisión de conocimientos, especialmente cuando no responden a las necesidades de los PeF, y basarnos en modelos que partan de las experiencias vividas por los profesores (enfoque experiencial), para que el modelo comience desde la persona misma y no desde el saber teórico. Muchos de los principios presentados son puntos básicos del aprendizaje realista (Korthagen 2001), que se basa a su vez en el enfoque reflexivo ${ }^{14}$, que Esteve y Alsina explican con las siguientes palabras:

Llamamos formación realista al planteamiento que apunta hacia la investigación de la persona con sus experiencias personales, los conocimientos teóricos, sus propias representaciones sobre lo que es enseñar y aprender, así como sus experiencias de aula (como aprendiz), y ello a través de la reflexión (Esteve y Alsina 2010: 10).

Dicho modelo, que es el que se debería aplicar en la formación de profesores ya desde los niveles iniciales, se denomina ALACT (Action, Looking back on the action, Awareness of essential aspects, Creating alternative methods of actions and Trial). El modelo, que fue presentado en el taller muy sucintamente a los asistentes, lo resume la misma autora en la siguiente figura:

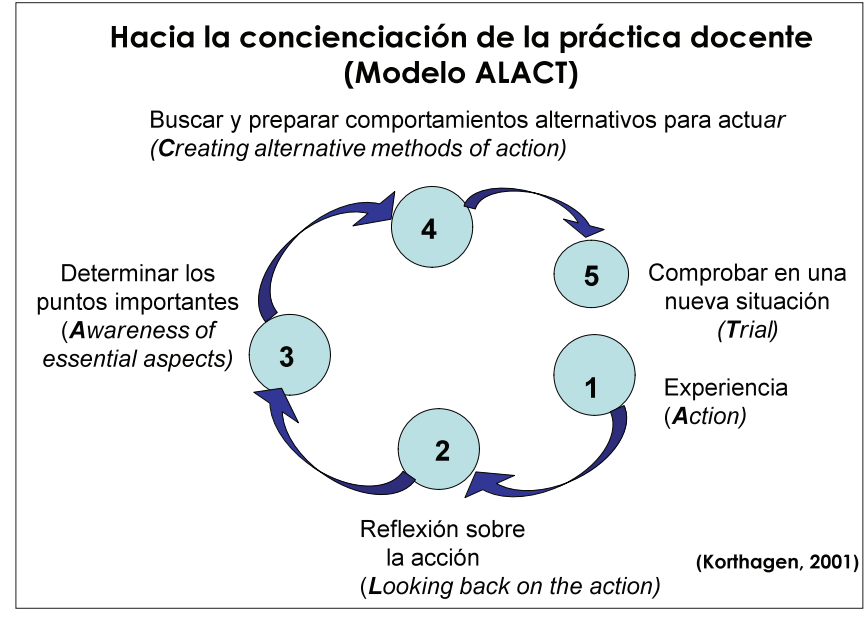

Figura 3.

14. Olga Esteve apunta que se basa en la teoría sociocultural de Vigotsky y lo define así: «El aprendizaje reflexivo se basa en una visión constructivista del aprendizaje (Freudenthal, 1991), según la cual el conocimiento sobre la práctica docente debe ser un conocimiento creado por el mismo sujeto en formación y no un conocimiento creado con anterioridad por terceros y transmitido por ellos. Es decir, la persona que se forma lo hace dando significado a unos contenidos, y no recibiendo unos contenidos ya impregnados de significado» (Esteve 2004: 10). 
Lo importante de este modelo es que concibe el aprendizaje como conexión entre las experiencias de los futuros profesores y el conocimiento teórico, que intercambia reflexión y acción y que no desprecia acudir a bases teóricas, pues reconoce que se puede recurrir a ellas entre la tercera y la cuarta fase, pero siempre para resolver un interrogante concreto, que haya surgido de la observación de un contexto determinado y que sea relevante para cada $\mathrm{PeF}$, no para algo que el formador de profesores haya decidido de antemano.

\section{El diseño, el desarrollo y la evaluación del taller formativo}

Detallamos a continuación los pasos que se siguieron para diseñar un taller de formación de profesores, impartido en el primer congreso EnELE, que pretendía ser un metataller que ilustrara y fuera consecuente con los principios metodológicos que acabamos de comentar. Para empezar, se realizó una propuesta formativa en la que los asistentes pudieron elegir la metodología; es decir, decidieron si querían asistir a una conferencia o participar en un taller. Dado que la mayoría se decidió por el taller, los $\mathrm{PeF}$ realizaron una serie de actividades, interactuaron con otros compañeros, reflexionaron, establecieron su punto de partida como docentes y evaluaron tanto la consecución de objetivos como la idoneidad de la metodología elegida.

La propuesta estaba diseñada para fomentar la reflexión sobre actitudes y creencias, para desarrollar ciertas habilidades docentes y para descubrir inductivamente los contenidos que hemos explicado en los epígrafes previos. Seguidamente analizamos las tres fases contempladas por Bernaus para la realización de actividades formativas (véase la figura 2). En el epígrafe siguiente se presentan algunos ejemplos de actividades formativas del IC, que también fueron analizadas en dicho taller.

\subsection{Antes de la formación}

Al recibir el encargo de impartir una conferencia sobre formación de profesores, teniendo en mente el principio 2.7, nos surgió la duda de si sería coherente defender un aprendizaje centrado en el alumno (principio 2.8) y orientado a la acción (principio 2.1) e impartir una conferencia, sobre todo porque se trataba de profesores que comenzaban su andadura profesional. Nuestra hipótesis de partida era que, si les permitíamos elegir la metodología que podía seguirse (bien una conferencia o bien un taller), la mayoría se decantaría por el taller y que, al evaluar esta decisión — si la actividad formativa se diseñaba apropiadamente y se ponían 
en práctica los principios metodológicos que se iban a explicar-, reconocerían las ventajas de aprender de forma reflexiva y a través de la acción.

La idea era recoger el principio metodológico 2.7, según el cual es más importante el ejemplo del profesor que sus propias ideas, opiniones y conocimientos, así como que los asistentes fueran conscientes de que una buena actividad formativa debe estar orientada a desarrollar toda la competencia docente, no solo los conocimientos, que son solo una parte de ella. Con la idea de promover el cambio en los PeF (principio 2.6) y de fomentar la reflexión (principio 2.4), se siguieron los tres pasos expuestos por Bernaus para la fase previa a la formación.

En primer lugar, perfilamos la idea de la que partíamos, para no limitarnos a transmitir conocimientos $y$, en segundo lugar, preguntamos a los organizadores por las necesidades de los asistentes al taller. Se confirmó que, en su mayoría, serían estudiantes de últimos años de grado y de máster de formación de profesores de español como lengua extranjera, sin apenas experiencia. En tercer lugar, para el diseño del taller, que esperábamos impartir si los asistentes elegían esa opción (aunque también se preparó la versión de la conferencia), se tomaron las siguientes decisiones, para aplicar los principios metodológicos comentados previamente:

- El principal objetivo del taller sería fomentar la reflexión y la capacidad crítica sobre cómo debe ser la formación (principio 2.4).

- Los contenidos serían pocos ${ }^{15}$ y se presentarían inductivamente, para que el PeF los dedujera a partir de las actividades. La información sobre contenidos o proyectos estaría subordinada a las actividades, no sería el foco y no se le dedicaría más de doce minutos del total de 90, pues era algo que se podía aprender fuera de la sesión. En el caso de que los PeF estuvieran interesados en profundizar en algún aspecto, se les ofrecerían unas cuantas referencias bibliográficas esenciales (principio 2.5).

+ Los asistentes tendrían la ocasión de elegir la metodología: no podíamos imponer esa innovación de convertir una conferencia en un taller, pues habría estado abocada al fracaso (principio 2.6). Además, se les pediría que reflexionaran sobre las razones de esa elección y, solo si la mayoría de los asistentes lo deseaban, se llevaría a cabo el experimento.

+ Se buscarían formas de recoger las opiniones de los asistentes, de modo que todos pudieran saber qué habían contestado los compañeros (principio 2.2). Por

15. Se hablaría de la evolución del concepto de competencia docente, de los dos proyectos en que participa el IC, así como de las líneas metodológicas para la formación de profesores. Se ilustraría con ejemplos de ciertas actividades formativas del IC y se aludiría a los documentos y proyectos europeos ya comentados: el PEFPI, el EPLTE y el QuailiTraining. 
ejemplo, se solicitaría a los asistentes que pegaran en una cartulina los post-it en que habían dibujado una metáfora sobre lo que para ellos era educar.

Se pensaría en algún sistema muy sencillo que permitiera evaluar la sesión y reflexionar sobre la idoneidad o no de haber convertido la conferencia en taller. Se entregaría al principio un post-it en el que se recogerían tres preguntas clave (al principio, a mitad y al final de la sesión) y se colocaría en una pared de la sala un cartel para que cada asistente pegara su post-it resumen de la experiencia:

- ¿Qué espero de esta sesión?

- ¿Qué opción he elegido: conferencia o taller? ¿Por qué?

- Conclusiones, ¿qué me llevo? ¿He hecho una buena elección?

+ El taller consistiría en una sucesión de actividades (principio 2.1) destinadas a fomentar un enfoque orientado a la acción y, siempre que fuera posible, se partiría del trabajo individual (que propiciara la reflexión), para después compartirlo en parejas o pequeños grupos y contrastarlo en gran grupo, para facilitar la reflexión individual y la interacción en grupos (principio 2.2). Se diseñaron nueve actividades y se tuvo en cuenta, como hacemos siempre en las clases de enseñanza de lenguas extranjeras, que hubiera variedad en los inputs que se presentaron y en la tipología de actividades. Unas actividades estaban más orientadas a la creación de un buen ambiente de trabajo, otras a la apropiación por parte del PeF de los objetivos y contenidos de la sesión, otra a la elección de la metodología, otra al establecimiento del punto de partida (principio 2.8), otras a la reflexión o bien a la aplicación de los conceptos aprendidos, así como también se incluyó alguna destinada a la evaluación del curso.

+ En esta experiencia formativa, concebida como metataller, se incluirían actividades que abordaran los componentes esenciales de toda actividad formativa (objetivos, contenidos, metodología y evaluación), garantizando que hubiera coherencia entre todos ellos (principio 2.7).

+ Lamentablemente, dado que era una sesión formativa muy breve, tuvimos que renunciar al importantísimo principio 2.3 (transferencia a su contexto), pues no había ocasión de realizar una práctica docente.

\subsection{Durante la actividad formativa}

Además de las actividades que ya se han comentado, nos gustaría destacar la inicial, que fue crucial para crear una imagen cercana de la ponente. Se eligió una actividad muy dinámica, que utilizara el juego y aportara cierta información personal, y que pusiera de pie a toda la sala para que los asistentes se adueñaran del espacio y no sintieran que lo dominaba la formadora. Se consiguió el objetivo de generar un clima de confianza, que animara a los estudiantes a probar algo 
nuevo. Tal y como se sugiere en la guía de Matei et alii (2007:33-35), es muy importante seleccionar cuidadosamente la actividad inicial, ya que es nuestra carta de presentación y en ella se plasman nuestras creencias sobre qué es y cómo debe ser una actividad formativa. Después, los PeF utilizaron la misma técnica para presentarse a sus compañeros.

Tras una breve explicación de los objetivos y contenidos de la sesión, esenciales para que los $\mathrm{PeF}$ supieran dónde pretendíamos llegar y qué iban a hacer y aprender en los siguientes minutos y pudieran autorregularse en la realización de las actividades (principio 2.5.) (Ferrer et alii 2012), se repartieron los post-it y se les pidió que escribieran qué esperaban de esa sesión y que lo compartieran con sus compañeros. A continuación, se pasó a la actividad en que se elegía la metodología y se votó si el grupo prefería una conferencia o un taller. Era una apuesta arriesgada, en un contexto poco favorable, pero, afortunadamente, la mayoría eligió taller; seguidamente se pusieron en común las razones de la elección, que resultaron muy enriquecedoras. Se insistió en que no había respuestas correctas ni incorrectas y se pidió a quienes habían optado por la conferencia que probaran la otra modalidad, pues posteriormente tendríamos la ocasión de reflexionar sobre si habíamos hecho o no una buena elección.

En cuanto al enfoque metodológico, nos decantábamos por el taller frente a la conferencia, porque permitía ilustrar mejor los principios metodológicos expuestos anteriormente y porque estaba más en consonancia con el enfoque metodológico de las actividades formativas del IC, que se resume en el siguiente párrafo: ${ }^{16}$

Desde un punto de vista metodológico, asumimos el carácter cognitivo, experiencial y constructivista del proceso de adquisición y aprendizaje de la competencia profesional del profesor de lenguas y somos conscientes de que, para que este proceso formativo sea eficaz y exitoso, es de vital importancia que las acciones de formación estén basadas en la acción, que partan de la observación y reflexión de la práctica docente, que ofrezcan oportunidades para experimentar e integrar teoría y práctica, y que exploten el potencial formativo que encierra la interacción con otros colegas y formadores.

Nos detenemos también en la actividad 6, en la que se pedía a los alumnos que reflexionaran sobre cómo aprendían mejor lenguas extranjeras (para retomar sus experiencias previas, tal y como defiende el aprendizaje realista), por qué y en qué contexto, a partir de unas dicotomías que servirían para presentar los principios metodológicos de la formación que íbamos a trabajar. Se insistió en que no había respuestas correctas ni incorrectas — cada alumno tendría una visión distinta, en función de sus creencias, de su estilo de aprendizaje y de su tradición

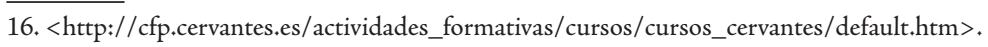


educativa- y en que la actividad pretendía hacer aflorar dichos valores y propiciar el debate, a sabiendas de que en algunas ocasiones los alumnos podrían elegir las dos opciones; en ese caso, lo importante sería el razonamiento para justificar cada opción en el momento de llevar a cabo la puesta en común. Después, los asistentes analizaron ejemplos de actividades formativas del IC, con los principios explicados. Nos detendremos en estos ejemplos en el epígrafe siguiente. La parte final del taller se dedicó a la evaluación. En primer lugar se llevó a cabo la actividad 8, en la que se volvieron a proyectar los objetivos de la sesión y se preguntó a los asistentes si se habían alcanzado; la respuesta unánime fue que sí, lo cual resulta decisivo, pues los asistentes deben salir de la actividad formativa con la idea de éxito y de haber cumplido lo que se pretendía conseguir. Para que esto suceda es muy importante que en la fase de diseño el profesor se pregunte qué pueden alcanzar los asistentes en ese corto periodo de tiempo formativo en el que trabajarán juntos y que en la fase de impartición de la sesión formativa haya una excelente gestión del tiempo, una habilidad docente muy difícil de alcanzar tanto para enseñar lengua como para formar profesores. Para poder completar adecuadamente la sesión y cerrar el ciclo, los $\mathrm{PeF}$ deben siempre disponer de un mínimo espacio de reflexión (principio 2.4) para elaborar y construir su aprendizaje: lo que se llevan de la sesión, cómo podrían aplicarlo, qué nuevas preguntas surgen y cómo pretenden darles respuesta, etc. (principio 2.5.). Solo así se puede producir un auténtico anclaje con sus conocimientos previos y puede haber transferencia a su contexto de enseñanza (principio 2.3).

Somos conscientes de que, stricto sensu, en una actividad formativa tan breve no se puede desarrollar la competencia docente, en el sentido de Perrenoud que hemos explicado en este artículo, pues haría falta observar a estos profesores en actuación y contrastar esa observación con su punto de partida. Sin embargo, sí pudimos reflexionar con ellos, en la actividad 9 sobre la metodología empleada en el taller, para comentar que, al menos, había ayudado a que la sesión no se centrara únicamente en conocimientos - aunque sí se presentaron algunos (el concepto de competencia docente, su evolución y algunos documentos europeos importantes)—, pues también se habían desarrollado habilidades: una técnica para comenzar una clase dinámica y generadora de un clima propicio para el trabajo en grupo; una manera de gestionar las interacciones de clase y de pedir silencio cuando se ha acabado el tiempo de debate; una mecánica de agrupamientos que va desde la reflexión individual al intercambio de aportaciones en pequeños grupos, para luego construir las respuestas entre todo el grupo; una técnica sencilla para verbalizar nuestras creencias a través de los dibujos en los post-it; una técnica para poner en común de forma rápida lo que han respondido los 
compañeros (pegándolo en un cartel), y una sencilla tarea para evaluar nuestra participación en el seminario, a través de las preguntas del post-it, que ayudaban a valorar el taller. En cuanto a las actitudes, los PeF apuntaron que se habían trabajado las de innovación, reflexión y capacidad crítica sobre cómo debe ser una actividad formativa y por qué, para saber también cómo debe ser la enseñanza de lenguas extranjeras, pues se basan en los mismos principios metodológicos. No se pudo completar esta actividad final, en la que se pedía a los $\mathrm{PeF}$ que reflexionaran sobre cuáles de los principios explicados se habían puesto en práctica en el taller, pero, a juzgar por sus respuestas en los post-it, creemos que la mayoría fue capaz de descubrir muchos de esos principios: reconocieron que el eje habían sido las actividades y no los contenidos (de hecho, el dosier era una sucesión de actividades); que se había fomentado la reflexión; que se había comenzado por su punto de partida y que había habido cierto espacio para la construcción conjunta del conocimiento a partir de la interacción con las actividades, el input, los compañeros y el formador; y también que había habido espacios para la reflexión individual y la elaboración y asimilación de lo experimentado.

En cuanto a la evaluación de los PeF, comentamos ahora algunas de las respuestas del post-it final. A la pregunta de si estaban satisfechos con la opción metodológica elegida, de los 30 post-it recogidos, 23 preferían el taller; 3, la conferencia; 3 una combinación de taller y conferencia, y una persona se mostraba dudosa. Se recogen aquí sus comentarios, agrupados por temas y destacados en negrita, pues ilustran las ventajas y la utilidad que han encontrado en el formato taller: 


\begin{tabular}{|c|c|c|}
\hline Preferencia & Comentario & $\begin{array}{c}\text { N.o } \\
\text { personas }\end{array}$ \\
\hline Taller & «Sesión más dinámica y entretenida» & 6 \\
\hline Taller & «Hemos aprendido más haciendo actividades». & 3 \\
\hline Taller & $\begin{array}{l}\text { «Más enriquecedora». «iGracias! Se cumplieron mis ex- } \\
\text { pectativas». }\end{array}$ & 4 \\
\hline Taller & $\begin{array}{l}\text { «Se aprende más mediante la reflexión». «Intentaré hacer } \\
\text { reflexionar a los alumnos y a la vez hacerlo yo misma». «Fo- } \\
\text { menta un aprendizaje práctico, autorreflexivo, en el que los } \\
\text { alumnos construyen sus propias definiciones y aplican pro- } \\
\text { cesos inductivos». }\end{array}$ & 3 \\
\hline Taller & $\begin{array}{l}\text { «Permite la interacción con los alumnos», «Se aprende más } \\
\text { compartiendo opiniones». «Ver la diferente paleta de colo- } \\
\text { res de opinión es gratificante». }\end{array}$ & 2 \\
\hline Taller & «Taller, porque el foco debe recaer en el alumno». & 1 \\
\hline Taller & «Para aprender cómo hacer un taller en un contexto así»». & 1 \\
\hline Taller & «Más adaptado para público no especializado». & 1 \\
\hline Taller & $\begin{array}{l}\text { «Porque uno perpetúa los métodos docentes que ha expe- } \\
\text { rimentado, no los que ha estudiado». }\end{array}$ & 1 \\
\hline Taller & $\begin{array}{l}\text { «Porque nos ha permitido experimentar aspectos que po- } \\
\text { dré aplicar en mis clases». }\end{array}$ & 1 \\
\hline Conferencia & $\begin{array}{l}\text { «Porque me siento más cómoda». (Al final esta alumna } \\
\text { dice: «Me ha gustado hacer un taller».) }\end{array}$ & 1 \\
\hline Conferencia & $\begin{array}{l}\text { «Si es una conferencia, elijo la conferencia». (Al final este } \\
\text { alumno dice: «No he perdido el tiempo haciendo un ta- } \\
\text { ller».) }\end{array}$ & 1 \\
\hline Conferencia & $\begin{array}{l}\text { «Porque quiero saber las opiniones de una experta». (Al } \\
\text { final este alumno diceः «Hay que combinar las dos opciones } \\
\text { de forma ecléctica».) }\end{array}$ & 1 \\
\hline Los dos & $\begin{array}{l}\text { «Conferencia, porque no tengo ganas de pensar, y taller } \\
\text { porque tengo ganas de interaccionar». «Equilibrio entre } \\
\text { opciones». }\end{array}$ & 3 \\
\hline Dudoso & $\begin{array}{l}\text { "Ha sido interesante y diferente como planteamiento de la } \\
\text { "conferencia", pero solo hemos planeado por las metodo- } \\
\text { logías». }\end{array}$ & 1 \\
\hline
\end{tabular}

Figura 4. 


\subsection{Después de la formación}

En cuanto a nuestra evaluación de la actividad formativa planteada — necesaria para reflexionar sobre ella, aprender y mejorarla-, es obvio que conlleva bastante tiempo secuenciar actividades, así como dosificar los contenidos y ubicarlos en el preciso lugar para que resulten asimilables por los PeF y sean relevantes para ellos; es decir, para que puedan relacionarlos con sus conocimientos previos, como sugiere el constructivismo. Sin embargo, somos conscientes de que es un tiempo bien empleado, pues estamos convencidos de que la experiencia activa y vital que supuso la actividad formativa perdurará mucho más tiempo que si se hubiera puesto el énfasis en los contenidos, los cuales, sin un anclaje en algo tangible, práctico y relacionado con la propia realidad y experiencia del alumno, se olvidarían en un brevísimo periodo de tiempo. Si deseamos ofrecer una formación significativa para el alumno, debemos partir de su realidad, apoyarnos en sus opiniones y creencias y ofrecer instrumentos para la reflexión y para resolver sus dudas.

Estos comentarios tan positivos y bastante entusiastas demuestran que la experiencia fue gratificante y satisfactoria para el grupo, que prefirió asistir a un taller incluso en ese contexto, que era una sucesión de conferencias, y también que los asistentes reconocen y han experimentado los beneficios de esta propuesta metodológica, lo cual confirma nuestra hipótesis inicial. Cabe preguntarse por la opinión de quienes no contestaron a esta actividad final, aproximadamente un tercio de los asistentes.

Como ya comentamos en el taller, habría sido necesario contar con otros noventa minutos y con un grupo más reducido, para que algunas de las conclusiones que comentamos en voz alta hubieran partido de los propios PeF. En otro contexto y en otras circunstancias, le habríamos dado prioridad a ese aspecto, si bien, como sugerencia de mejora, constatamos que habría sido una buena idea eliminar alguna actividad para dedicar más tiempo a cada una de ellas.

\section{Algunos ejemplos de buenas prácticas}

Finalizamos el artículo explicando la actividad 7, en la que los PeF dispusieron de tiempo para analizar en pequeños grupos siete ejemplos de actividades formativas del IC, que ilustraban varios de los principios metodológicos explicados en el epígrafe 2 y que ellos ya habían descubierto inductivamente. El primero era un ejemplo de nuestros cursos en línea, más concretamente del Nivel inicial para profesores de español (de 140 horas de duración), en el que se aprecia que hay una sucesión de actividades (enfoque orientado a la acción) y, por tanto, que los 
contenidos están supeditados a la reflexión inductiva y activa que supone la realización de dichas actividades (como defiende el aprendizaje realista: alejarse del enfoque deductivo, que parte de la teoría para aplicarla después a la práctica). Se fomenta la autonomía del alumno, pues este decide si realiza primero las actividades interactivas en que se descubren los contenidos de forma inductiva o bien si lee primero los contenidos, en el documento «contenidos imprimibles». Por otra parte, los títulos del módulo («El punto de partida de mi desarrollo profesional») y de la unidad formativa ( «De mi experiencia como aprendiente a las bases de mi desarrollo profesional» $)^{17}$ nos acercaban a la realidad del alumno, lo cual es un exponente del enfoque reflexivo y del aprendizaje realista. También se comentó que la tarea evaluativa de este curso es la elaboración del punto de partida del portfolio, lo que ponía de manifiesto la importancia otorgada a la reflexión y a la autonomía.

El segundo ejemplo correspondía precisamente a un ejemplo de esos portfolios, que permitía apreciar cómo se fomentaba la reflexión del PeF sobre lo que hace, por qué lo hace y si lo modificaría después de contrastarlo con la teoría y con las opiniones comentadas en los foros y en otras actividades de comunicación. Por todo ello, era un claro ejemplo del enfoque reflexivo.

El tercer ejemplo era el programa del Máster Universitario en Enseñanza de Español como Lengua Extranjera IC-UIMP, ${ }^{18}$ que se diseñó teniendo en cuenta los descriptores del EPLTE.

\begin{tabular}{|c|c|c|c|c|}
\hline \multicolumn{5}{|c|}{$\begin{array}{l}\text { Programa Máster Universitario en Enseñanza de Español } \\
\text { como Lengua Extranjera IC-UIMP }\end{array}$} \\
\hline $\begin{array}{l}\text { Bloque lectivo I } \\
\text { (16 créditos) }\end{array}$ & $\begin{array}{l}\text { Practicum } 1 \\
\text { (6 créditos) }\end{array}$ & $\begin{array}{l}\text { Practicum II } \\
\text { (12 créditos) }\end{array}$ & $\begin{array}{c}\text { Bloque lectivo } \\
\text { I I } \\
\text { (14 créditos) }\end{array}$ & $\begin{array}{c}\text { Trabajo fin } \\
\text { de máster (12 } \\
\text { créditos) }\end{array}$ \\
\hline $\begin{array}{l}\text { Periodo lectivo } \\
\text { de introducción } \\
\text { a los contenidos } \\
\text { teórico-prácticos } \\
\text { fundamentales para el } \\
\text { profesor de español. } \\
\text { Incluye un módulo de } \\
\text { observación de clases } \\
\text { (Módulo de aula). }\end{array}$ & $\begin{array}{l}\text { Primer periodo } \\
\text { de prácticas } \\
\text { autorizadas. }\end{array}$ & $\begin{array}{l}\text { Segundo } \\
\text { periodo de } \\
\text { experiencia } \\
\text { docente. }\end{array}$ & $\begin{array}{l}\text { Periodo } \\
\text { lectivo de } \\
\text { profundización } \\
\text { en lingüística } \\
\text { aplicada a la } \\
\text { enseñanza de } \\
\text { español y de } \\
\text { desarrollo de } \\
\text { habilidades } \\
\text { docentes. }\end{array}$ & $\begin{array}{l}\text { Trabajo de } \\
\text { investigación } \\
\text { centrado en } \\
\text { algún aspecto de } \\
\text { la enseñanza de } \\
\text { ELE y basado } \\
\text { en la experiencia } \\
\text { docente propia. }\end{array}$ \\
\hline
\end{tabular}

Figura 5.

17. Tanto los cursos presenciales como los cursos en línea se estructuran en unidades formativas, entendidas como unidades de programación breves y completas que tienen unos objetivos, unos contenidos y una evaluación. 18. Véase <http://cfp.cervantes.es/actividades_formativas/master/default.htm>. 
Se destacaron los siguientes aspectos:

+ Enfoque orientado a la acción: se integra teoría y práctica docente, porque el objetivo es aprender a actuar con competencia. También se aprecia en el desarrollo de la competencia docente que se propone en todas sus dimensiones: el Bloque lectivo I se centra más en contenidos; en el Módulo de observación y en los Practicum I y II se pretende el desarrollo de habilidades y actitudes, y en el Bloque lectivo II, finalmente, se trabajan habilidades docentes.

+ Aprendizaje realista: el trabajo de fin de máster se debe basar en la experiencia docente propia del alumno, para que se pueda concretar la transferencia al contexto del alumno.

+ Las clases presenciales de los bloques lectivos se imparten en formato taller, para permitir la construcción conjunta del conocimiento, basada en los principios del constructivismo y del interaccionismo social.

El cuarto ejemplo era un extracto del portfolio de una alumna del máster ICUIMP (promoción 2008-2009) que se seleccionó para ilustrar cómo aprenden los PeF de la observación de su actuación docente (enfoque reflexivo), ya que durante el Practicum II los PeF grababan una clase, lo que les permitía analizar y reflexionar sobre si actuaban con competencia (enfoque orientado a la acción, que aplica el nuevo concepto de competencia docente) y comentar tanto los aspectos positivos como los mejorables. Con la ayuda del tutor de prácticas y de los coordinadores de prácticas del IC o de la UIMP, se guía al alumno en ese proceso reflexivo; sin embargo, es el alumno quien realiza realmente ese aprendizaje.

[... T Tras la observación de la grabación de clase me he dado cuenta de que hablo demasiado. Parece que evito los silencios, que me resultan incómodos o son síntoma de que algo no funciona. Nunca antes había notado esto. Creo que puede deberse al nivel del curso y a la dificultad de obtener feedback de los alumnos acerca de su comprensión y seguimiento de las actividades y contenidos. Parece que interpreto siempre de manera negativa estos silencios, sin dar tregua al alumno, que quizá necesite más tiempo de reflexión. Me parece conveniente ofrecer un input rico al alumno, pero cuando tiene un sentido y una finalidad concreta. En este caso, doy demasiados ecos, repito muchas partículas de confirmación que pueden distraer al alumno de su tarea. También soy consciente de que se ha tratado de un grupo (por su contexto y tradición educativa) que exigía mucha motivación, modelos y ejemplos; sin embargo, creo que hablo más de lo que debería.

Figura 6. 
El quinto ejemplo era una actividad de comunicación de un curso en línea, que permitía fomentar el aprendizaje por interacción entre iguales, defendido por el aprendizaje realista, como buen heredero del constructivismo en el que se basa.

El sexto ejemplo correspondía al programa de un curso de formación de profesores del IC, concretamente el 263 - Formación de profesores de español (nivel B), que se impartió en el año $2011^{19}$ y en el que se podía apreciar que las actividades formativas partían del punto en el que se encontraba el alumno. Asimismo, era un buen ejemplo del enfoque realista, por el diseño del componente práctico y del enfoque reflexivo, y porque se realizaba una carpeta o portfolio, en el que el alumno dejaba constancia de su progreso, lo cual constituyó el ejemplo 7, el último de los que se analizaron en el taller.

\section{Conclusiones}

En este artículo se han revisado algunos principios metodológicos de la formación de profesores de lenguas extranjeras y se ha explicado cómo se diseñó, desarrolló y analizó una experiencia formativa presentada en el primer congreso EnELE, que pretendía ser un ejemplo de cómo fomentar la interacción y la reflexión de los $\mathrm{PeF}$, para que pudieran sacar el máximo provecho de esa experiencia, que es irrepetible, en tanto en cuanto con otros PeF los resultados habrían sido distintos. El grupo que participó en esta experiencia aprovechó plenamente las actividades formativas que se apoyan en los enfoques metodológicos que se han explicado en estas páginas, lo cual confirma nuestra hipótesis de que los $\mathrm{PeF}$ sí están preparados para recibir una formación aplicada y práctica, que siga los supuestos del aprendizaje realista. Finalizamos estas líneas con la cita con la que cerramos el taller: «Dime cómo enseñas y te diré cómo piensas que aprenden tus alumnos» (Martínez 2012).

19. La información completa de este curso puede encontrarse en <http://www.cervantes.es/lengua_y_ensenanza/cursos_formacion_profesores/cfp_181-09.htm>. 


\section{Referencias bibliográficas}

Bernaus, M. (2012): «European documents and their implications for language teacher trainers: TRAIN-ED», en DiAdori, P. (ed.), How to Train Language Teacher Trainers, Newcastle upon Tyne, Cambridge Scholars Publishing.

Bolitho, R. y Wright, T. (1997): «Working with participants' ideas and constructs», en McGrath, I. (ed.), Learning to Train: Perspectives on the Development of Language Teacher Trainers, Hemel Hempstead, Prentice-Hall.

Consejo de Europa (2002): Marco común europeo de referencia para las lenguas: aprendizaje, enseñanza, evaluación, Madrid, Anaya ( $1 .^{a}$ ed. en 2001) [Disponible en: <http://cvc.cervantes.es/ensenanza/biblioteca_ele/marco/>].

Diadori, P. (ed.) (2010): Formazione. Qualità. Certificazione, Verona, Le Monnier.

- (2012): How to Train Language Teacher Trainers, Newcastle upon Tyne, Cambridge Scholars Publishing, en prensa.

Esteve, O. (2004): «Nuevas perspectivas en la formación del profesorado de lenguas: hacia el "aprendizaje reflexivo" o "aprender a través de la práctica"», en Actas de las Jornadas Didácticas de Español y Alemán como Lenguas Extranjeras, Bremen, CVC. [Disponible en: <http://cvc.cervantes.es/ensenanza/ biblioteca_ele/publicaciones_centros/bremen_2004.htm>].

Esteve, O. y Alsina, A. (2010): «Hacia el desarrollo de la competencia profesional del profesorado», en Esteve, O.; Melief, K. y Alsina, A., Creando mi profesión. Una propuesta para el desarrollo profesional del profesorado, Barcelona, Octaedro.

Esteve, O.; Melief, K.y Alsina, A. (2010): Creando mi profesión. Una propuesta para el desarrollo profesional del profesorado, Barcelona, Octaedro.

Ferrer, À.; Navajas, A.; Herrera, J:; Menéndez, G. y Rivas, S. (2012): «Trabajar con objetivos de aprendizaje (I)», DidactiRed [Disponible en: $<$ http://cvc.cervantes.es/aula/didactired/anteriores/abril_12/23042012. htm $>$ ].

Instituto Cervantes (2004): Marco general del Instituto Cervantes para la formación de profesores de español como lengua extranjera y como segunda lengua, Madrid, inédito.

- (2006): Plan curricular del Instituto Cervantes. Niveles de referencia para el espanol, Madrid, Biblioteca Nueva.

- (2012). Las competencias clave del profesorado de lenguas segundas y extranjeras, Madrid, Instituto Cervantes. 
Kelly, M.; Grenfell, M.; Allan, R.; Kriza, Ch. y McEvoy, W. (2004)ः European Profile for Language Teacher Education. A Frame of Reference, Southampton, University of Southampton.

Kelly, M. (2012): «European documents and their implications for language teacher trainers: The European Profile for Language Teacher Education - A Frame of Reference (EPLTE)», en Diadori, P. (ed.), How to Train Language Teacher Trainers, Newcastle upon Tyne, Cambridge Scholars Publishing.

Komorowska, H. (2012): «European documents and their implications for language teacher trainers: The European Portfolio for Student Teachers of Languages (EPOSTL)», en Diadori, P. (ed.), How to Train Language Teacher Trainers, Newcastle upon Tyne, Cambridge Scholars Publishing.

Korthagen, F. (2001): Linking Practice and Theory. The Pedagogy of Realistic Teacher Education, London, Lawrence Erlbaum Associates.

Mann, S. (2005). «The language teacher development», en Language Teaching, 38, Cambridge University Press: 103-118. [Disponible en: <http //www2. warwick.ac.uk/fac/soc/al/staff/teaching/mann/mann_s/stateof.pdf $>$ ].

Martín Peris, E. (2009): «La formación inicial en el marco del desarrollo de la competencia docente», ponencia en el curso El profesor de segundas lenguas II: La formación inicial y el desarrollo de la competencia docente, Colección IFB, vol. 66 (I), Donostia, Universidad del País Vasco.

Martínez Aldanondo, J. (2012): Gestión del conocimiento, núm. 70, Catenaria. [Disponible en: <http://www.catenaria.cl $/ \mathrm{km} /$ newsletter/newsletter_70.htm >].

Matei, G.; Bernaus, M.; Heyworth, K.; Powl, U. y Wright, T. (2007): First steps in teacher training. A practical guide. The TrainEd kit, Strasbourg, Council of Europe, European Centre for Modern Languages. [Disponible en: <http://www.ecml.at/mtp2/Trained/html/TrainED_E_Results.htm>].

Muresan, L.; Heyworth, F; Mateva, G. y Rose, M. (2007): QualiTraining - A Training Guide for Quality Assurance in Language Education, Strasbourg, Council of Europe.

Muresan, L. (2012): «European documents and their implications for language teacher trainers: QuAlitraining», en Diadori, P. (ed.), How to Train Language Teacher Trainers, Newcastle upon Tyne, Cambridge Scholars Publishing.

Newby, D;; Allan, R.; Fenner, A.; Jones, B.; Komorowska, H. y SoghikYAN, K. (2007): Portfolio europeo para futuros profesores de idiomas, Strasbourg, 
Council of Europe, European Centre for Modern Languages [Disponible en: <http://www.ecml.at/mtp2/FTE/pdf/STPExtract.pdf>].

Norti, B*; Mateva, G. y Rossner, R. (2007/2009)ः A Profiling Grid for Language Teachers, EAQUALS, v. 0.30 [Disponible en: <http://www.eaquals. org/pages $/ \mathrm{p}=7104>$ ].

Perrenoud, P. (2001): «La formación de los docentes en el siglo XXI», Revista de Tecnología Educativa, XIV, 3: 503-523.

- (2004): Diez nuevas competencias para enseñar, Barcelona, Graó.

Rodrigo, C. (2011): De la transmisión de conocimientos al desarrollo de la competencia profesional de lenguas: análisis de una práctica reflexiva, Madrid, Universidad Politécnica de Madrid, trabajo DEA inédito.

Rossner, R. (2010): «Una griglia di descrittori per il profile professionale dei docenti di lingue: uno strumento per lo sviluppo professionale e per la gestione della formazione permanente», en Diadori, P. (ed), Formazione. Qualità. Certificazione, Verona, Le Monnier.

Thorne, K. y Mackey D. (2003): Everything you ever needed to know about training, London, Kogan Page.

Verdía, E. (2010): «De la adquisición del conocimiento al desarrollo de la competencia docente: profesionalización de los profesores de ELE», en Actas de los II Encuentros en Comillas El profesor de ELE: metodología, técnicas y recursos para el aula [Disponible en: <http://www.encuentroselecomillas.es/archivos/Actas_Encuentros_ELE_2010_B.pdf $>$ ].

- (2012): «La formación de profesores en el Instituto Cervantesः de los cursos de formación al desarrollo de estándares para profesores», Anuario del Instituto Cervantes 2012, Madrid, Instituto Cervantes: 265-282.

VV.AA. (2011-2013): «European Profiling Grid» [Disponible en: <www.epgproject.eu> ].

VV.AA. (2012): «Qué significa ser un profesor de ELE competente», Taller virtual en el I congreso mundial COMPROFES [Disponible en: < http://comprofes.es/talleres $>$ ]. 


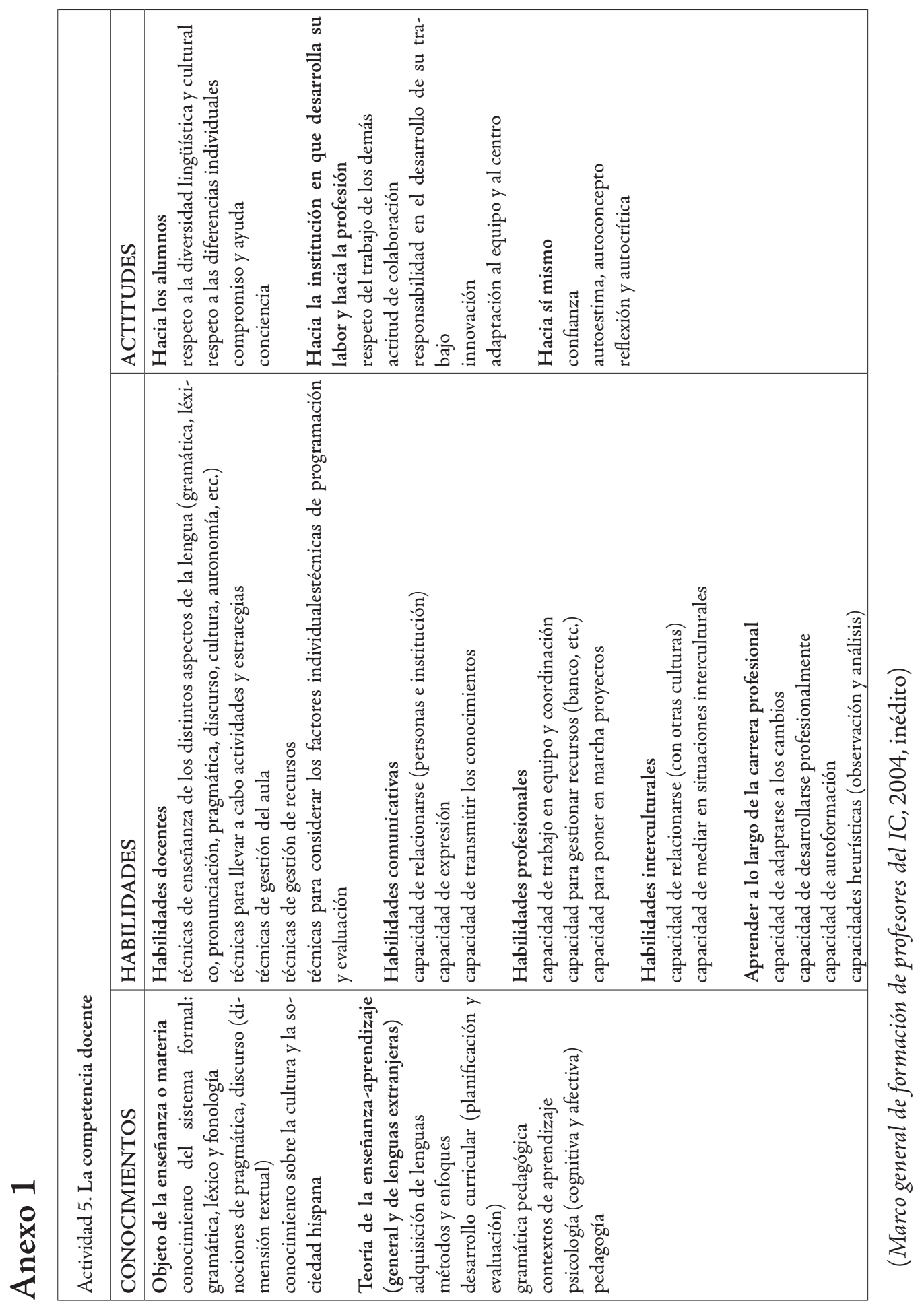




\section{Anexo 2}

\section{MODELO DE COMPETENCIAS CLAVE DEL PROFESORADO DE LENGUAS SEGUNDAS Y EXTRANJERAS}

\section{COMPETENCIAS CLAVE}

\section{a) Organizar situaciones de aprendizaje.}

b) Evaluar el aprendizaje y la actuación del alumno.

c) Implicar a los alumnos en el control de su propio aprendizaje.

d) Facilitar la comunicación intercultural.

e) Desarrollarse profesionalmente como profesor de la institución.

f) Gestionar sentimientos y emociones en el desempeño de su trabajo.

g) Participar activamente en la institución.

h) Servirse de las TIC para el desempeño de su trabajo.

\section{COMPETENCIAS ESPECÍFICAS}

$>$ Diagnosticar y atender las necesidades de los alumnos.

$>$ Promover el uso y la reflexión sobre la lengua.

$>$ Planificar secuencias didácticas.

$>$ Gestionar el aula.

$>$ Servirse de herramientas y procedimientos de evaluación.

$>$ Garantizar buenas prácticas en la evaluación.

$>$ Promover una retroalimentación constructiva.

$>$ Implicar al alumno en la evaluación.

$>$ Promover que el alumno gestione los recursos y medios disponibles para aprender.

> Integrar en la enseñanza herramientas para reflexionar sobre el proceso de aprendizaje.

$>$ Promover que el alumno defina su propio proyecto de aprendizaje.

> Motivar al alumno para que se responsabilice de su propio aprendizaje.

Implicarse en el desarrollo de la propia competencia intercultural.

$>$ Adaptarse a las culturas del entorno.

Fomentar el diálogo intercultural.

Promover que el alumno desarrolle su competencia intercultural.

$>$ Analizar y reflexionar sobre la práctica docente.

$>$ Definir un plan personal de formación continua.

$>$ Implicarse en el desarrollo profesional del equipo docente.

$>$ Participar activamente en el desarrollo de la profesión.

$>$ Gestionar las propias emociones.

$>$ Motivarse en el trabajo.

$>$ Desarrollar las relaciones interpersonales.

$>$ Implicarse en el desarrollo de la inteligencia emocional del alumno.

Trabajar en equipo en el centro.

Implicarse en los proyectos de mejora del centro.

Promover y difundir buenas prácticas en la institución.

Conocer la institución e integrarse en ella.

$>$ Implicarse en el desarrollo de la propia competencia digital.

$>$ Desenvolverse en entornos digitales y con aplicaciones informáticas disponibles.

Aprovechar el potencial didáctico de las TIC.

Promover que el alumno se sirva de las TIC para su aprendizaje.

(Instituto Cervantes, 2012: 11) 